Check for updates

Cite this: RSC Adv., 2018, 8, 5967

Received 19th December 2017

Accepted 29th January 2018

DOI: 10.1039/c7ra13460f

rsc.li/rsc-advances

\section{Investigation on enhanced photocatalytic degradation of bisphenol A with bismuth oxyiodide catalyst using response surface methodology $\dagger$}

\begin{abstract}
Dan Zhang, Fei Wang, (iD * Suzhen Cao and Xiaoli Duan*
In this study, $\mathrm{Bi}_{7} \mathrm{O}_{9} \mathrm{I}_{3}$ photocatalyst was successfully synthesized via a simple and rapid microwave irradiation method. The characterization of prepared photocatalysts was determined by powder X-ray diffraction (XRD), X-ray photoelectron spectroscopy (XPS), scanning electron microscopy (SEM), transmission electron microscopy (TEM), energy dispersive spectrometry (EDS), UV-vis diffuse reflectance spectroscopy (DRS) and photoluminescence (PL). The photocatalytic performance was determines by the degradation of bisphenol A (BPA) under xenon lamp illumination. The $\mathrm{Bi}_{7} \mathrm{O}_{9} \mathrm{I}_{3}$ catalyst exhibited superior photocatalytic performance and the first-order kinetic rate constant of $\mathrm{Bi}_{7} \mathrm{O}_{9} \mathrm{I}_{3}$ was about 4.2 times greater than that of $\mathrm{BiOl}$. The enhanced photocatalytic activity was associated with surface morphology, suitable band gap energy and low recombination rate of electron-hole pairs. Furthermore, the photocatalytic efficiency of BPA with $\mathrm{Bi}_{7} \mathrm{O}_{9} l_{3}$ was systematically investigated using a three factor, three level Box-Behnken experimental design and response surface methodology (RSM). A quadratic polynomial model was proposed. Experimental and predicted values exhibited a good correlation with a predicted $R^{2}$ value of 0.9016 . A relative significance study of three independent variables showed that catalyst dosage had the most significant positive effect on the degradation of BPA, followed by initial concentration of $\mathrm{BPA}$ and $\mathrm{pH}$ value. The prepared $\mathrm{Bi}_{7} \mathrm{O}_{9} \mathrm{I}_{3}$ is a promising photocatalyst for practical application in organic pollutant decomposition.
\end{abstract}

\section{Introduction}

Bisphenol A (2,2-(4,4-dihydroxydiphenyl)propane, BPA) is extensively used in the synthesis of polycarbonate, epoxy resins, coating materials and other consumer products. ${ }^{1}$ The global consumption of BPA was estimated to be over 5.5 million metric tons in $2011 .^{2} \mathrm{BPA}$ is likely released into the environment not only by discharge from synthesis, but also from migration, usage and disposal methods. Hence, BPA has been detected in various environmental media, including municipal wastewaters, sewage, surface water, landfills and so on. ${ }^{3,4}$ Moreover, previous experimental and epidemiological studies $^{5,6}$ have shown that BPA had the potential to affect the endocrine system through disturbing the synthesis, transport and metabolism of estrogenic and thyroid hormones. BPA has caused great concern due to its widespread application and large production.

School of Energy and Environmental Engineering, University of Science and Technology Beijing, Xueyuan Road No. 30, Haidian District, Beijing 100083, PR China. E-mail: wangfei@ustb.edu.cn; jasmine@ustb.edu.cn

$\uparrow$ Electronic supplementary information (ESI) available: Experimental levels for Box-Behnken design (Table S1), adequacy summary of the models (Table S2), TEM images (Fig. S1), FT-IR spectra (Fig. S2), EDS spectra (Fig. S3), plots of $\ln \left(C_{t} / C_{0}\right)$ versus reaction time (Fig. S4), PL spectra (Fig. S5), $\mathrm{N}_{2}$ adsorption-desorption isotherm (Fig. S6), plots of predicted values versus actual values and residuals (Fig. S7). See DOI: 10.1039/c7ra13460f
Therefore, effective removal techniques are required, such as sorption, chemical oxidation and biodegradation. In particular, there is increasing interest in photocatalytic technology because of its effective activity and low toxicity. ${ }^{-10}$

Recently, bismuth oxyiodides $\left(\mathrm{Bi}_{x} \mathrm{O}_{y} \mathrm{I}_{z}\right)$ have attracted extensive attentions as a family of promising visible-light-responsive photocatalysts. ${ }^{11-15}$ The remarkable photocatalytic property is associated with their unique $\mathrm{Bi}$ 6s orbital and anisotropic layered structure, ${ }^{16,17}$ which generates the internal electric field perpendicular to each layer and then facilitates to separate the photogenerated electrons and holes. ${ }^{18}$ Previous studies on $\mathrm{Bi}_{7} \mathrm{O}_{9} \mathrm{I}_{3}$ (ref. 19-23) have indicated its excellent photocatalytic activity and promising prospect in environmental decontamination. Among them, few studies ${ }^{18}$ focused on microwave irradiation synthesis of $\mathrm{Bi}_{7} \mathrm{O}_{9} \mathrm{I}_{3}$, which has the advantage of short reaction time, homogeneity, high product yield and purity. ${ }^{16,24}$ In addition, research into exploring the relative significance of different degradation parameters, such as catalyst dosage, pollutant concentration, $\mathrm{pH}$ value, on its photocatalytic activity is limited.

Response surface methodology (RSM) has been proved to be a powerful tool for studying the influence of parameters and their interactions on the experimental system..$^{25,26}$ For experimental design, Box-Behnken design (BBD) is widely used for RSM due to the advantage of estimation parameters of the 
quadratic model, sequential design building and detection the model by lack of fit. ${ }^{27}$ BBD and other response surface designs have been applied to solve drawbacks and optimize procedures in a variety of analytical techniques, such as extraction processes, sorption processes and analytical separations..$^{28-30}$ However, very few studies reported the application of response surface modelling to investigate the influence factors in photocatalytic degradation process by $\mathrm{Bi}_{7} \mathrm{O}_{9} \mathrm{I}_{3}$.

In the present study, facile and fast microwave irradiation approach was attempted to synthesize $\mathrm{Bi}_{7} \mathrm{O}_{9} \mathrm{I}_{3}$ photocatalyst. The structure, chemical state, morphology, composition and optical absorption property were characterized over $\mathrm{Bi}_{7} \mathrm{O}_{9} \mathrm{I}_{3}$ and compared with as-synthesized BiOI. Then, $\mathrm{Bi}_{7} \mathrm{O}_{9} \mathrm{I}_{3}$ was employed for the photocatalytic degradation of BPA under xenon lamp illumination. To get a better understanding of the effects of independent variables such as catalyst dosage, initial concentration of BPA and $\mathrm{pH}$ value on removal efficiency of BPA over $\mathrm{Bi}_{7} \mathrm{O}_{9} \mathrm{I}_{3}$ photocatalyst, response surface methodology based on Box-Behnken experimental design was applied and an empirical quadratic response model was proposed. Analysis of variance (ANOVA) and response surface analysis were utilized to explore relative significance of variables and their interactions. Combined RSM-BBD approach in this study shows the feasibility to provide effective information on photocatalytic reaction condition for BPA removal.

\section{Experimental}

\subsection{Materials and reagents}

Bismuth nitrate pentahydrate $\left(\mathrm{Bi}\left(\mathrm{NO}_{3}\right)_{3} \cdot 5 \mathrm{H}_{2} \mathrm{O}\right)$, potassium iodide (KI) and bisphenol A (BPA) were purchased from Fuchen Chemical Reagents Factory (China). Ethanol, methanol and ethylene glycol (EG) were provided by Sinopharm Chemical Reagent Co., Ltd (China). The Degussa $\mathrm{P}_{25} \mathrm{TiO}_{2}$ was obtained from Degussa Corporation (Germany). All other reagents were analytical grade and were used without further purification. Deionized water was used to prepare the experimental solutions. $0.1 \mathrm{M} \mathrm{HCl}$ and $2 \mathrm{M} \mathrm{NaOH}$ solutions were used to adjust $\mathrm{pH}$ values.

\subsection{Microwave irradiation synthesis of the photocatalysts}

The photocatalysts were synthesized by a facile microwave irradiation method. $1.5 \mathrm{mmol} \mathrm{Bi}\left(\mathrm{NO}_{3}\right)_{3} \cdot 5 \mathrm{H}_{2} \mathrm{O}$ and $1.5 \mathrm{mmol} \mathrm{KI}$ were orderly dissolved in $35 \mathrm{~mL}$ ethylene glycol with continuous magnetic stirring and $\mathrm{pH}$ value was adjusted to 9 by adding $2 \mathrm{M}$ $\mathrm{NaOH}$. Subsequently, the obtained solution was transferred into a Teflon vessel and then performed in a microwave chemical reactor (COOLPEX, Preekem Scientific Instruments Co., Ltd, China) for synthesis reaction. The reaction was conducted at $160{ }^{\circ} \mathrm{C}$ for $30 \mathrm{~min}$. Then, formed product was collected by centrifugation after the temperature dropped down to $50{ }^{\circ} \mathrm{C}$. The precipitate was washed several times with deionized water and ethanol, respectively. Finally, the obtained $\mathrm{Bi}_{7} \mathrm{O}_{9} \mathrm{I}_{3}$ sample were dried at $60^{\circ} \mathrm{C}$ in an oven. For comparison, BiOI sample was synthesized using the same process without $\mathrm{pH}$ adjustment.

\subsection{Photocatalytic experiments}

The photocatalytic degradation experiments were conducted in a photochemical reactor (YZ-GHX-A, China) equipped with a $500 \mathrm{~W}$ xenon lamp and with circulating water system to maintain the reaction isothermally. $100 \mathrm{~mL}$ of BPA aqueous solutions with various initial concentrations (from $10 \mathrm{mg} \mathrm{L}^{-1}$ to $50 \mathrm{mg} \mathrm{L}^{-1}$ ) were prepared and mixed with photocatalyst powder ranging from $0.1 \mathrm{~g} \mathrm{~L}^{-1}$ to $2.0 \mathrm{~g} \mathrm{~L}^{-1}$ under magnetic stirring. Then reaction solutions were stirred in the dark for $30 \mathrm{~min}$ in order to ensure adsorption/desorption equilibrium prior to irradiation. Photocatalytic tests were performed at $20 \pm 1{ }^{\circ} \mathrm{C}$ and at different time intervals, $1 \mathrm{~mL}$ of the suspension was withdrawn and centrifuged ( $8000 \mathrm{rpm}, 10 \mathrm{~min}$ ) to remove the solids. The obtained supernatants were stored at $4{ }^{\circ} \mathrm{C}$ before concentration analysis.

\subsection{Instruments and analytical methods}

The phase structure of as-synthesized samples was determined by a powder X-ray diffraction (XRD, Ultima IV, Rigaku Corp., Japan) with $\mathrm{Cu}-\mathrm{K} \alpha$ irradiation at 2 theta range of $10-90^{\circ}$. Transmission electron microscopy (TEM) was determined on a FEI Tecnai G2 F20 TEM (FEI, USA). The chemical state of elements in the samples was detected by X-ray photoelectron spectroscopy (XPS, Escalab 250Xi, Thermo Fisher Scientific, USA). Fourier transform infra-red (FT-IR) measurements were conducted on a Nexus 410 spectrometer (Nicolet, USA). The surface morphologies of samples were investigated by a field emission scanning electron microscopy (FE-SEM, JSM 6701F, JEOL, Japan) and elemental mapping was analyzed by an energy dispersive spectrometer (EDS). UV-vis diffuse reflectance spectra (DRS) of obtained samples were performed on a UV-vis spectrophotometer (TU-1901, Persee General Corp., China), where $\mathrm{BaSO}_{4}$ was used as the reference. The detected wavelength ranged from $300 \mathrm{~nm}$ to $800 \mathrm{~nm}$ and the reflectance was converted into absorption according to Kubenka-Munk method. Photoluminescence (PL) measurements were conducted at an excitation wavelength of $290 \mathrm{~nm}$ using a Hitachi F7000. The Brunauer-Emmett-Teller (BET) specific surface area of $\mathrm{Bi}_{7} \mathrm{O}_{9} \mathrm{I}_{3}$ sample $\left(S_{\mathrm{BET}}\right)$ was measured with ASAP 2460 (Micrometrics, USA) and nitrogen gas was used as the adsorbate at liquid temperature. The concentration of BPA was analyzed using high-performance liquid chromatography (HPLC, LC20AD, Shimadzu, Japan) with UV-vis detector. Athena C18-WP column $(4.6 \mathrm{~mm} \times 250 \mathrm{~mm}, 5 \mu \mathrm{m}, \mathrm{CNW})$ was used for separation and the mobile phase was consisted of methanol and water at a ratio of $80: 20(\mathrm{v} / \mathrm{v})$. The flow rate was set as $0.7 \mathrm{~mL} \mathrm{~min}^{-1}$ and detection wavelength was chosen at $224 \mathrm{~nm}$.

\subsection{Experimental design and statistical analysis}

Design Expert 8.0 software (Stat-Ease, Inc., USA) was used to design experiments and analyze mathematical modeling. BoxBehnken design (BBD) was applied in this study because of its rotatable or nearly rotatable second-order design. ${ }^{31}$ Three independent variables composed of catalyst dosage, initial concentration of BPA and pH value. Each factor was coded to 
three levels $(-1,0$ and +1$)$, which represented for low, central and high level, respectively. Experimental ranges and levels of each independent variable were shown in ESI Table S1. $\dagger$ Experimental data designed by BBD were analyzed to fit a second-order polynomial equation, which aimed to estimate the mathematical relationship between independent variables and response. ${ }^{32}$ The general form of quadratic response model was formulated as eqn (1). ${ }^{33}$

$$
\begin{aligned}
Y= & \beta_{0}+\beta_{1} A+\beta_{2} B+\beta_{3} C+\beta_{11} A^{2}+\beta_{22} B^{2}+\beta_{33} C^{2}+\beta_{12} A B \\
& +\beta_{23} B C+\beta_{31} C A
\end{aligned}
$$

where $Y$ is the predicted response, $\beta_{0}$ is the model intercept coefficient, $\beta_{1}, \beta_{2}$ and $\beta_{3}$ represent for coefficients of independent variables, $\beta_{11}, \beta_{22}$ and $\beta_{33}$ are model quadratic coefficients, $\beta_{12}, \beta_{23}$ and $\beta_{31}$ are interactive regression coefficients, respectively and $A, B, C$ indicated three independent variables investigated.

The analysis of variance (ANOVA) was used to approximate statistical relationship based on the proposed model and examine results of experimental design. The coefficient of determination $\left(R^{2}\right)$ estimated the fitting quality of regression mode ${ }^{25}$ and response surface analysis was conducted to visualize individual and interactive influences of three variables on the photocatalytic degradation of BPA.

\section{Results and discussion}

\subsection{Characterization of the as-synthesized photocatalysts}

The phase structure of as-obtained bismuth oxyiodide samples was characterized by XRD. As shown in Fig. 1(a), diffractive pattern of sample without $\mathrm{pH}$ adjustment is in accordance with tetragonal structure of BiOI (JCPDS no. 10-0445) in the region of 10-90 ${ }^{\circ}$ For synthesized sample at $\mathrm{pH} 9$, diffraction peaks seem similar to that of BiOI with slightly shift, but it could not be indexed to an existed standard JCPDS card for bismuth containing phase. Main diffraction peaks centered at $28.7^{\circ}, 31.5^{\circ}$, $36.6^{\circ}, 45.2^{\circ}, 49.0^{\circ}$ and $54.4^{\circ}$ are displayed to match well with characteristic diffraction angles of $\mathrm{Bi}_{7} \mathrm{O}_{9} \mathrm{I}_{3}$ according to previous studies. ${ }^{18-20,22,34,35}$ Hence, the sample is tentatively identified as $\mathrm{Bi}_{7} \mathrm{O}_{9} \mathrm{I}_{3}$. In addition, no other characteristic peaks of bismuth oxides, basic bismuth nitrate or impurities are presented in Fig. 1(a). It indicates that two as-synthesized samples are both consisted of high-purity phase. Meanwhile, strong peaks are indicative of well crystallized samples and the intensity of diffraction peaks for $\mathrm{Bi}_{7} \mathrm{O}_{9} \mathrm{I}_{3}$ becomes relatively higher than that of BiOI, which is likely associated with improved crystallinity of $\mathrm{Bi}_{7} \mathrm{O}_{9} \mathrm{I}_{3}$. Besides, slightly shift in diffraction angles between $\mathrm{Bi}_{7} \mathrm{O}_{9} \mathrm{I}_{3}$ and $\mathrm{BiOI}$ is probably related to extra bismuth and oxygen atoms in the lattice leading to expansion and distortion of standard BiOI structure. ${ }^{19}$ The formation of $\mathrm{Bi}_{7} \mathrm{O}_{9} \mathrm{I}_{3}$ and $\mathrm{BiOI}$ suggests that $\mathrm{pH}$ value of microwave irradiation synthesis solution plays important role in the composition of bismuth oxyiodide products. It has been concluded that BiOI was generated at the beginning and subsequent substitution of I by $\mathrm{OH}^{-}$was gradually proceeded with increased $\mathrm{pH}$ value in alkaline conditions, contributing to reduced iodine contents. $^{36,37}$ Additionally, the TEM images of as-synthesized BiOI and $\mathrm{Bi}_{7} \mathrm{O}_{9} \mathrm{I}_{3}$ samples were displayed in Fig. S1. $\uparrow$ Two sets of different lattice images are observed with a $d$-spacing of $0.282 \mathrm{~nm}$ and $0.317 \mathrm{~nm}$, corresponding to the (1 10 ) plane of $\mathrm{BiOI}^{15}$ and the $\left(\begin{array}{lll}1 & 1 & 0\end{array}\right)$ plane of $\mathrm{Bi}_{7} \mathrm{O}_{9} \mathrm{I}_{3},{ }^{23}$ respectively. The TEM result is consistent with the $\mathrm{XRD}$ analysis.

The chemical states of two samples were investigated by XPS and high resolution XPS spectra of $\mathrm{Bi} 4 \mathrm{f}$, I $3 \mathrm{~d}$ and $\mathrm{O} 1 \mathrm{~s}$ were displayed in Fig. 1(b)-(d), respectively. BiOI and $\mathrm{Bi}_{7} \mathrm{O}_{9} \mathrm{I}_{3}$ are observed to have similar binding energies of characteristic peaks at the Bi and I region (Fig. 1(b) and (c)). The two peaks of
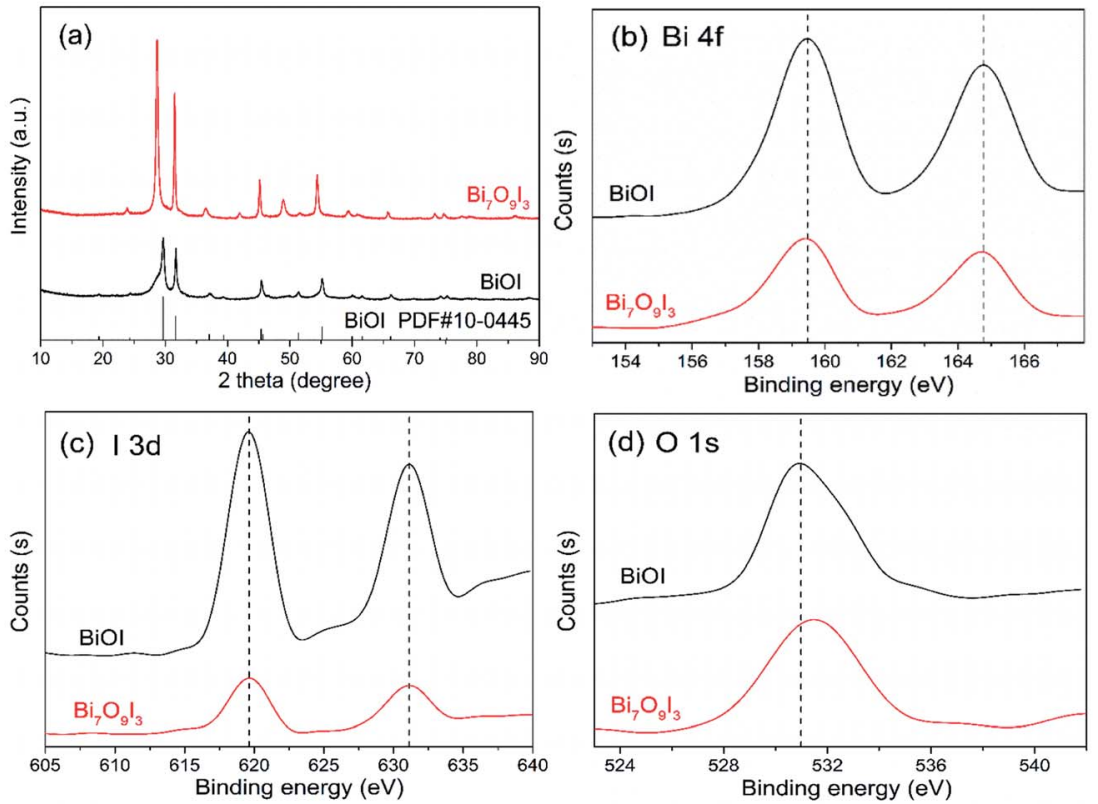

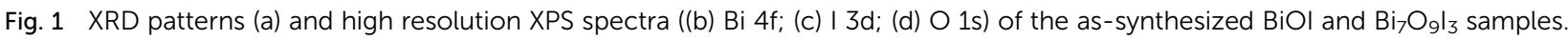



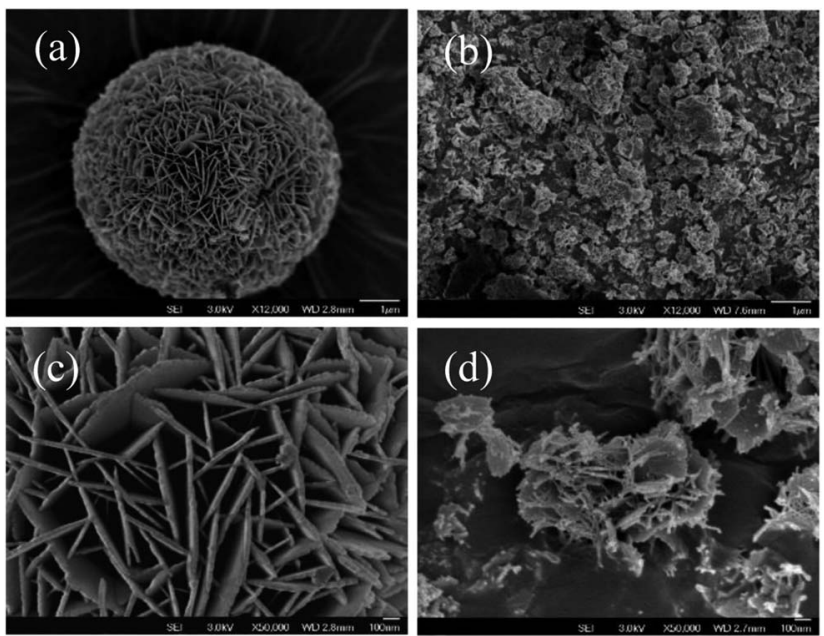

Fig. 2 SEM images of as-synthesized $\mathrm{BiOl}$ and $\mathrm{Bi}_{7} \mathrm{O}_{9} \mathrm{I}_{3}$ samples at low magnification ( $a$ and $b$ ) and high magnification ( $c$ and $d$ ).

159.4 $\mathrm{eV}$ and $164.7 \mathrm{eV}$ (Fig. 1(b)) are corresponded to Bi $4 \mathrm{f}_{7 / 2}$ and $\mathrm{Bi} 4 \mathrm{f}_{5 / 2}$, respectively, indicating trivalent state for bismuth element in two samples. ${ }^{3,39}$ The I $3 \mathrm{~d}_{5 / 2}$ and I $3 \mathrm{~d}_{3 / 2}$ peaks (Fig. 1(c)) locating at $619.6 \mathrm{eV}$ and $631.1 \mathrm{eV}$ are ascribed to monovalence state of $\mathrm{I}^{21}$ According to Fig. 1(d), slightly different $\mathrm{O} 1 \mathrm{~s}$ spectra between $\mathrm{BiOI}$ and $\mathrm{Bi}_{7} \mathrm{O}_{9} \mathrm{I}_{3}$ are likely associated with the chemisorbed $\mathrm{H}_{2} \mathrm{O}$ or $\mathrm{HO}^{-}$on the surface. ${ }^{20}$ The FT-IR spectra of two samples were shown in Fig. S2, $\uparrow$ where the absorption bands located in the range of $400-700 \mathrm{~cm}^{-1}$ indicated the stretching vibrations of $\mathrm{Bi}-\mathrm{O}$ and $\mathrm{Bi}-\mathrm{O}-\mathrm{I}$ in bismuth oxyiodides. ${ }^{13}$ The absorption at $1630 \mathrm{~cm}^{-1}$ and $3400 \mathrm{~cm}^{-1}$ is likely attributed to the hydrogen-bond hydroxyl groups and physically absorbed water molecules. ${ }^{\mathbf{4 0 , 4 1}}$

The surface morphology of prepared photocatalysts were characterized by FE-SEM-EDS, as shown in Fig. 2(a)-(d). In comparison with SEM images at low ( $a$ and b) and high (c and d) magnification, $\mathrm{pH}$ value of as-synthesized reaction is observed to have significant influence on the shape and size of obtained products. For BiOI sample with no addition of $\mathrm{NaOH}$ (Fig. 2(a) and (c)), it is clearly shown micro-flower structure, which is assembled by interlaced nanosheets. The sheet size of microsphere structure is in the range of micrometers and high magnified SEM image displays plenty of irregular nanosheets with a length of several hundred nanometers. In contrast, no apparent flowerlike structure is observed in the surface of $\mathrm{Bi}_{7} \mathrm{O}_{9} \mathrm{I}_{3}$ sample (pH 9, Fig. 2(b) and (d)) and the highmagnification image shows irregular nanoflakes and thin nanorods. In addition, EDS result (Fig. S3†) exhibits that elemental composition of two samples are both $\mathrm{Bi}, \mathrm{O}$ and $\mathrm{I}$. The obtained atomic ratio of $\mathrm{Bi}$ to $\mathrm{I}$ is $1: 1.03$ and $1: 0.42$, respectively, which is near to the stoichiometric ratio of BiOI and $\mathrm{Bi}_{7} \mathrm{O}_{9} \mathrm{I}_{3}$ within experimental error. Therefore, the result provides further evidence on the composition of as-synthesized samples and is in accordance with previous analysis of XRD patterns.

DRS was applied to investigate the optical properties of assynthesized $\mathrm{BiOI}$ and $\mathrm{Bi}_{7} \mathrm{O}_{9} \mathrm{I}_{3}$ samples. As shown in Fig. 3(a),
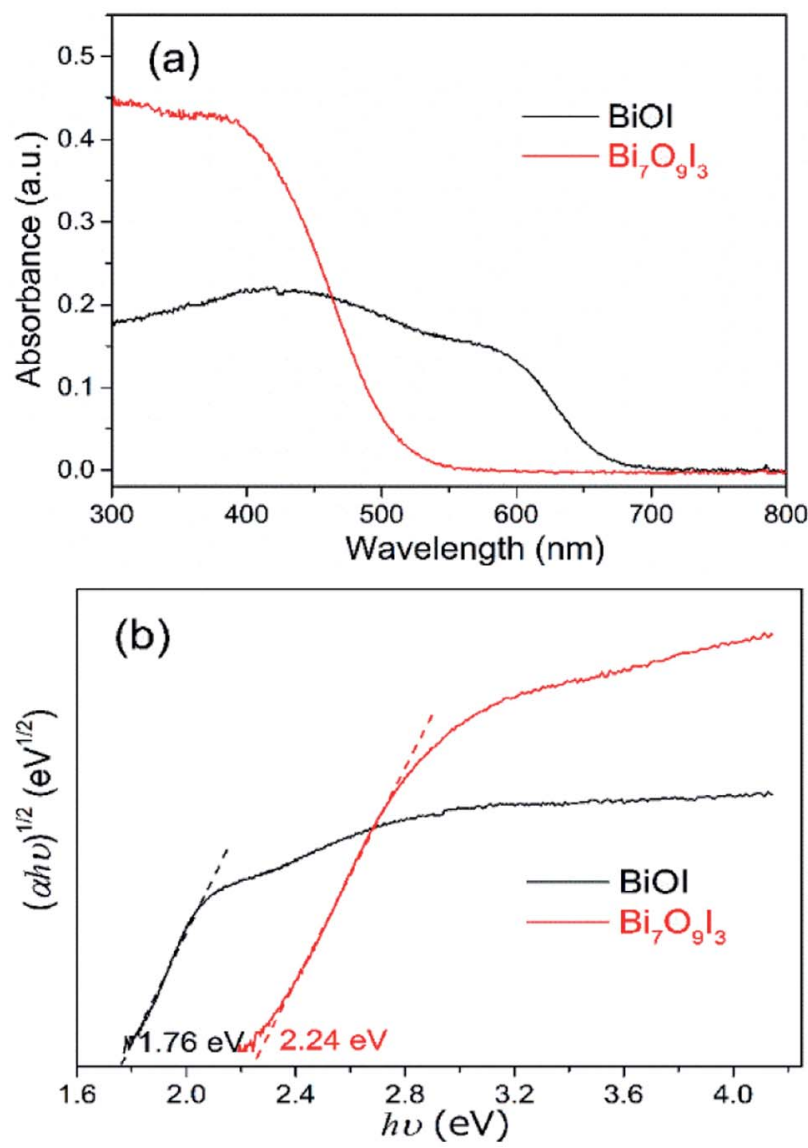

Fig. 3 UV-vis diffuse reflectance spectra (a) and plots of $(\alpha h \nu)^{1 / 2} v s . h \nu$ (b) of as-synthesized $\mathrm{BiOl}$ and $\mathrm{Bi}_{7} \mathrm{O}_{9} l_{3}$.

two samples both exhibit photoabsorption from ultraviolet to visible light range. The obtained absorption edge of $\mathrm{Bi}_{7} \mathrm{O}_{9} \mathrm{I}_{3}$ sample is approximately $510 \mathrm{~nm}$ and that of BiOI is found to be about $667 \mathrm{~nm}$, indicating that optical absorption properties of bismuth oxyiodides vary with chemical composition. Moreover, optical band gap energy for semiconductor materials can be determined by the equation: ${ }^{42} \alpha h \nu=A\left(h \nu-E_{\mathrm{g}}\right)^{n / 2}$, where $\alpha, h, \nu$, $A$ and $E_{\mathrm{g}}$ are absorption coefficient, Planck constant, light frequency, a constant and band gap energy, respectively. The value of parameter $n$ depends on the optical transition type of a semiconductor. For direct transition, $n$ equals to 1 , whereas the value of $n$ is 4 for indirect transition, such as Bi-based oxyiodides. Therefore, band gap energy of as-synthesized samples is estimated by extrapolating the straight line from plot of $(\alpha h \nu)^{1 / 2}$ versus photon energy $(h \nu)$ to $X$ axis, as shown in Fig. 3(b). The calculated $E_{\mathrm{g}}$ of $\mathrm{BiOI}$ and $\mathrm{Bi}_{7} \mathrm{O}_{9} \mathrm{I}_{3}$ is approximately $1.76 \mathrm{eV}$ and $2.24 \mathrm{eV}$, respectively, which is consistent with relevant reported values in previous studies. ${ }^{\mathbf{1 8 , 2 2 , 4 3 , 4 4}}$ In addition, $\mathrm{Bi}_{7} \mathrm{O}_{9} \mathrm{I}_{3}$ sample is observed to have a higher $E_{\mathrm{g}}$ value compared to BiOI and it is likely associated with reduced stoichiometric proportion of I.

\subsection{Photocatalytic degradation of BPA}

The photocatalytic activity of as-synthesized samples was evaluated by the degradation of BPA (a typical endocrine disrupting 


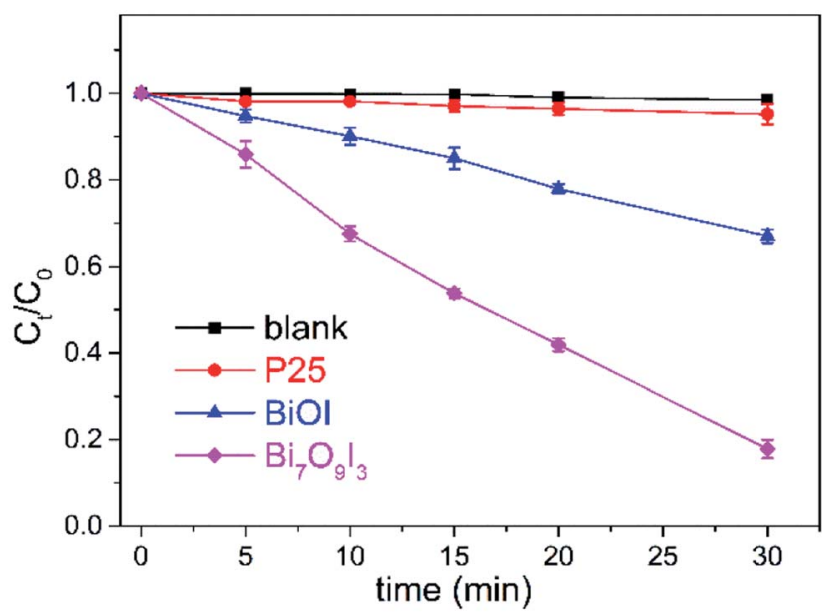

Fig. 4 Photocatalytic degradation of BPA in the presence of different photocatalysts. In some cases, error bars are smaller than the symbols.

contaminant) under xenon lamp illumination. The variations of BPA concentration with irradiation time over blank, Degussa $\mathrm{P} 25$, BiOI and $\mathrm{Bi}_{7} \mathrm{O}_{9} \mathrm{I}_{3}$ were displayed in Fig. 4. The degradation of BPA in the absence of photocatalyst (blank) could be neglected due to almost constant concentration during the whole irradiation process. In contrast, $\mathrm{Bi}_{7} \mathrm{O}_{9} \mathrm{I}_{3}$ sample exhibits the highest photocatalytic activity than Degussa P25 and BiOI under identical irradiation conditions. Degradation efficiency of BPA achieved $82 \%$ with $0.5 \mathrm{~g} \mathrm{~L}^{-1} \mathrm{Bi}_{7} \mathrm{O}_{9} \mathrm{I}_{3}$ at initial BPA concentration of $20 \mathrm{mg} \mathrm{L}^{-1}$ after irradiation within $30 \mathrm{~min}$, while 5\% and 33\% degradation occurred over P25 and BiOI, respectively. The results indicate an outstanding photocatalytic capability of $\mathrm{Bi}_{7} \mathrm{O}_{9} \mathrm{I}_{3}$ for BPA decomposition.

To further investigate the degradation kinetic of BPA by different photocatalysts, pseudo first-order kinetic equation (eqn (2) $)^{45}$ was applied to fit experimental data.

$$
\ln \frac{C_{t}}{C_{0}}=-k t
$$

where $C_{t}$ and $C_{0}$ correspond to reactant concentration at time $t$ and 0 , respectively, $k$ is the first-order rate constant and $t$ stands for reaction time.

The plots of $\ln \left(C_{t} / C_{0}\right)$ versus $t$ (Fig. S4 $\dagger$ ) suggest that photocatalytic degradation of BPA fits well by pseudo first-order kinetic with good correlation coefficients. The calculated $k$ values for $\mathrm{P} 25, \mathrm{BiOI}$ and $\mathrm{Bi}_{7} \mathrm{O}_{9} \mathrm{I}_{3}$ are $0.0015,0.0134$ and $0.0565 \mathrm{~min}^{-1}$, respectively, reflecting the maximum rate constant of $\mathrm{Bi}_{7} \mathrm{O}_{9} \mathrm{I}_{3}$ for BPA degradation. In addition, $k$ value of $\mathrm{Bi}_{7} \mathrm{O}_{9} \mathrm{I}_{3}$ is observed to be about 4.2 time greater than that of BiOI. The photocatalytic performance of catalysts is considered to be related with the efficient separation of electron-hole pairs. ${ }^{36,46}$ PL spectra of $\mathrm{BiOI}$ and $\mathrm{Bi}_{7} \mathrm{O}_{9} \mathrm{I}_{3}$ samples were determined to explore the recombination rate of the photogenerated electron-hole pairs ${ }^{47}$ and the results are presented in Fig. S5. $\dagger$ PL spectra of both samples are observed to be similar and one main emission peak appears at about $470 \mathrm{~nm}$. However, the PL intensity of $\mathrm{Bi}_{7} \mathrm{O}_{9} \mathrm{I}_{3}$ is lower than that of BiOI. The weaker PL intensity generally corresponds to the higher possibility of
Table 1 Coded Box-Behnken design matrix along with experimental and predicted values for removal efficiency (\%) of $\mathrm{BPA}$ by $\mathrm{Bi}_{7} \mathrm{O}_{9} \mathrm{I}_{3}$

\begin{tabular}{|c|c|c|c|c|c|}
\hline \multirow[b]{2}{*}{ Run } & \multicolumn{3}{|c|}{ Coded levels } & \multicolumn{2}{|c|}{ Removal efficiency (\%) of BPA } \\
\hline & $A$ & $B$ & $C$ & Experimental & Predicted \\
\hline 1 & 1 & 0 & -1 & 44.59 & 45.25 \\
\hline 2 & -1 & 1 & 0 & 6.90 & 5.14 \\
\hline 3 & 0 & 0 & 0 & 49.93 & 48.36 \\
\hline 4 & -1 & -1 & 0 & 26.40 & 29.95 \\
\hline 5 & 0 & 1 & 1 & 48.04 & 50.47 \\
\hline 6 & 0 & -1 & 1 & 89.70 & 86.82 \\
\hline 7 & -1 & 0 & -1 & 6.15 & 5.02 \\
\hline 8 & -1 & 0 & 1 & 13.05 & 12.39 \\
\hline 9 & 1 & 0 & 1 & 72.42 & 73.54 \\
\hline 10 & 0 & 1 & -1 & 26.88 & 29.77 \\
\hline 11 & 0 & 0 & 0 & 45.22 & 48.36 \\
\hline 12 & 0 & -1 & -1 & 74.29 & 71.87 \\
\hline 13 & 1 & -1 & 0 & 93.29 & 95.06 \\
\hline 14 & 0 & 0 & 0 & 49.94 & 48.36 \\
\hline 15 & 1 & 1 & 0 & 44.97 & 41.42 \\
\hline
\end{tabular}

photogenerated carrier separation. ${ }^{39}$ Therefore, the results indicate that the recombination rate of electron-hole pairs for $\mathrm{Bi}_{7} \mathrm{O}_{9} \mathrm{I}_{3}$ is effectively inhibited, thus leading to the enhancement of photocatalytic degradation performance.

As shown in Fig. S6, $\dagger$ the nitrogen adsorption-desorption isotherm of $\mathrm{Bi}_{7} \mathrm{O}_{9} \mathrm{I}_{3}$ sample is close to Type IV with a $\mathrm{H} 3$ hysteresis loop. ${ }^{48,49}$ The isotherm curve indicates the existence of split-like pores, which are generally generated by the aggregation of plate-like particles..$^{50,51}$ This result is in agreement with the FE-SEM result. The BET surface area of $\mathrm{Bi}_{7} \mathrm{O}_{9} \mathrm{I}_{3}$ is measured to be $18.90 \mathrm{~m}^{2} \mathrm{~g}^{-1}$ and has the potential to supply a large number of surface active sites..$^{52}$ Thus, the BET value play a role in the superior photocatalytic activity of $\mathrm{Bi}_{7} \mathrm{O}_{9} \mathrm{I}_{3}$ sample. Therefore, $\mathrm{Bi}_{7} \mathrm{O}_{9} \mathrm{I}_{3}$ photocatalyst is used in the following study.

During the photocatalytic degradation processes, various primary active species, such as holes, hydroxyl radicals ( $\left.{ }^{\circ} \mathrm{OH}\right)$, superoxide radicals $\left(\mathrm{O}_{2}{ }^{-\cdot}\right)$ and singlet oxygen, could be formed in the UV-vis/semiconductor systems. ${ }^{23,46}$ The standard redox potential of $\mathrm{Bi}^{5+} / \mathrm{Bi}^{3+}$ is more negative than that of ${ }^{\circ} \mathrm{OH} / \mathrm{OH}^{-} .{ }^{36}$ Thus, photogenerated holes on the surface of bismuth oxyiodides are not preferred to react with $\mathrm{OH}^{-} / \mathrm{H}_{2} \mathrm{O}$ to generate ${ }^{\circ} \mathrm{OH},{ }^{47}$ which indicates that the degradation of BPA is likely associated with the direct reaction with the photogenerated hole or with $\mathrm{O}_{2}{ }^{-\cdot}$ (generated by the excited electron) or both species.

\subsection{Model fitting and statistical analysis}

Box-Behnken design consisting of 15 experimental runs was performed to investigate the influence of three independent variables on the degradation efficiency of BPA. Design matrix with experimental and predicted response were presented in Table 1. To evaluate the adequacy of various models, different tests including sequential model sum of squares, lack of fit tests and model summary statistic were carried out. Quadratic model is considered as most suitable model among linear, 2FI, quadratic and cubic models, because it exhibits maximum $R^{2}$, 
adjusted $R^{2}$ and predicted $R^{2}$ values excluding cubic model which is aliased, as shown in Table S2. $\dagger$

Therefore, quadratic model was further analyzed through analysis of variance (ANOVA). An empirical second order polynomial equation (eqn (3)) was estimated between response and variables following by statistical analysis on experimental data.

$$
\begin{aligned}
Y= & 48.36+25.35 A-19.61 B+8.91 C-15.58 A^{2}+10.10 B^{2} \\
& +1.26 C^{2}-7.21 A B+1.44 B C+5.23 C A
\end{aligned}
$$

where $Y$ is the removal efficiency at 20 min reaction, $A, B$ and $C$ represent coded values of catalyst dosage, initial concentration of BPA and $\mathrm{pH}$ value, respectively. The positive sign before a term in eqn (3) represents an interactive effect of the variable, whereas a negative sign indicates an antagonistic effect. ${ }^{53}$

Detailed results of quadratic model fitting in ANOVA analysis were presented in Table 2. The large Fisher $(F)$ value (75.09) and corresponding $p$-value $(<0.0001)$ for estimated model indicate that the model is significant, because $p$-value (Prob $>F$ ) less than 0.05 is considered to demonstrate a statistical significant model term. There is only a $0.01 \%$ chance that a model $F$-value occurs due to noise. In addition, lack of fit (LOF) indicates data variation around proposed model and is always considered as a special adequacy test if model fits. ${ }^{53}$ The $F$-value of LOF (2.86) and corresponding $p$-value of 0.2700 imply that LOF is not significant relative to pure error. Non-significant LOF is expected for a model to predict response successfully.

According to the calculated $F$-values and $p$-values of each model terms (Table 2), three independent variables $(A, B$ and $C$ ), interactive parameter between $A$ (catalyst dosage) and $B$ (initial concentration of BPA), interaction term between $A$ and $C$ (pH value) and two quadratic coefficients $\left(A^{2}\right.$ and $\left.B^{2}\right)$ are statistically significant due to small $p$-values less than 0.05. Influential order of three variables on the removal efficiency of BPA is $A$ (catalyst dosage) $>B$ (initial concentration of $\mathrm{BPA})>C$ (pH value). Therefore, catalyst dosage is hypothesized to be the most important factor in this study for BPA photocatalytic decomposition by $\mathrm{Bi}_{7} \mathrm{O}_{9} \mathrm{I}_{3}$, which is corresponded to the highest $F$ value (328.99) and lowest $p$-value $(<0.0001)$. This finding is supported by the fact that degradation efficiency would increase significantly with increasing catalyst dosage due to larger

Table 2 ANOVA for removal efficiency of BPA from Box-Behnken design

\begin{tabular}{lrrrll}
\hline Source & Sum of squares & df & Mean square & $F$ value & $p$-Value \\
\hline Model & 10558.21 & 9 & 1173.13 & 75.09 & $<0.0001$ \\
$A$ & 5139.86 & 1 & 5139.86 & 328.99 & $<0.0001$ \\
$B$ & 3076.99 & 1 & 3076.99 & 196.95 & $<0.0001$ \\
$C$ & 635.58 & 1 & 635.58 & 40.68 & 0.0014 \\
$A B$ & 207.75 & 1 & 207.75 & 13.30 & 0.0148 \\
$A C$ & 109.45 & 1 & 109.45 & 7.01 & 0.0456 \\
$B C$ & 8.29 & 1 & 8.29 & 0.53 & 0.4991 \\
$A^{2}$ & 895.72 & 1 & 895.72 & 57.33 & 0.0006 \\
$B^{2}$ & 376.85 & 1 & 376.85 & 24.12 & 0.0044 \\
$C^{2}$ & 5.91 & 1 & 5.91 & 0.38 & 0.5655 \\
Residual & 78.12 & 5 & 15.62 & & \\
Lack of fit & 63.33 & 3 & 21.11 & 2.86 & 0.2700
\end{tabular}

amount of catalytic active sites when the dosage is within optimal value. ${ }^{54}$ The negative influence of BPA concentration can be explained that more organic molecules would be absorbed on the surface of catalyst when initial concentration of organic compound increases, which inhibits the photogeneration of reactive species resulting from overloading of the active sites. In addition, another likely explanation is the increasing amount of incident photons which are absorbed by the organic molecules prior to reach the catalyst. ${ }^{37}$ Among linear interactions, $\mathrm{pH}$ value shows the least effect on the model and squared terms of $C^{2}$ is found to be not significant with the largest $p$-value of 0.5655 .

The coefficient of determination $\left(R^{2}\right)$ was calculated with the ratio of explained variation to total variation and was considered as an effective indicator to evaluate fitting degree of proposed model. $R^{2}$ values in the empirical regression equation by ANOVA analysis were calculated and determination coefficient $\left(R^{2}=0.9927\right)$ value of the quadratic model is close to adjusted determination coefficient (Adj- $\left.R^{2}=0.9794\right)$. It indicates that the model is appropriate, because the value of Adj- $R^{2}$ would become noticeably smaller than $R^{2}$ if there are many terms and sample size is not large enough in the model..$^{55} \mathrm{In}$ addition, coefficient of variance (CV) is used to indicate the reproducibility of a model and a model with $\mathrm{CV}$ value smaller than $10 \%$ was considered as reasonably reproducible. ${ }^{56} \mathrm{Ob}-$ tained $\mathrm{CV}$ value of $8.57 \%$ seems plausible to support acceptable deviation between experimental and estimated values. Parameter of adequate precision (AP) measures the signal to noise ratio, and a value higher than 4 is desirable to favor the model. The calculated AP value of 27.897 indicates an adequate signal and this model is feasible to navigate the design space. Moreover, the value of $\operatorname{Adj}-R^{2}$ is in agreement with predicted $R^{2}$ value (0.9016), indicating a good correlation between experimental and predicted values (removal efficiency), as shown in Table 1. The plots of predicted values versus observed data were presented in Fig. S7a $\dagger$ and acceptable agreement is observed. Normal probability of internally studentized residuals was plotted in Fig. S7b. $\dagger$ No serious violation is observed underlying the analyses and normality assumption is confirmed with approximately a straight line. In summary, statistical analysis results by ANOVA indicate that quadratic polynomial model is appropriate to study the influence of independent variables and predict photocatalytic degradation of BPA in the experimental range.

\subsection{Response surface analysis}

Three-dimensional (3D) response surface plots and contour plots were graphical represented in Fig. 5 over three independent variables $(A, B$ and $C)$ and attempted to explore the relationship of two variables when the third variable was fixed a constant value at central level. The combined effect of catalyst dosage and initial BPA concentration on response at a constant $\mathrm{pH}$ value (pH 6.5) was shown in Fig. 5(a1) and (a2). The shapes of contour plots are recommended to provide information on the nature and extent of the interactions. A contour plot with elliptical curves is likely associated with prominent interactions 


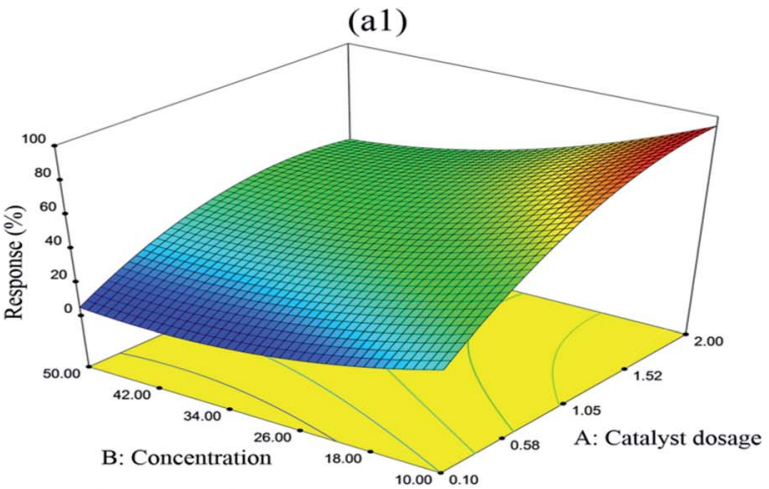

(b1)

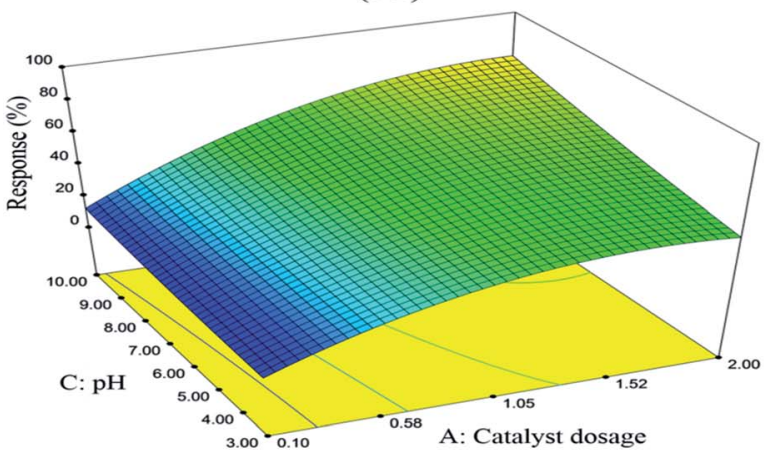

(c1)

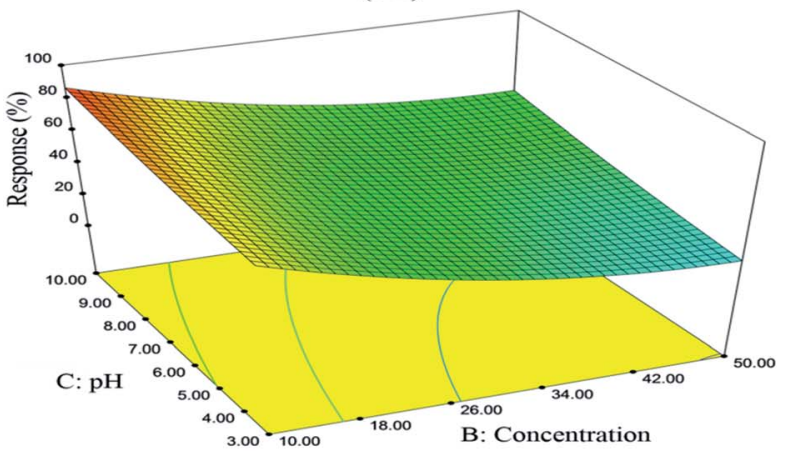

(a2)

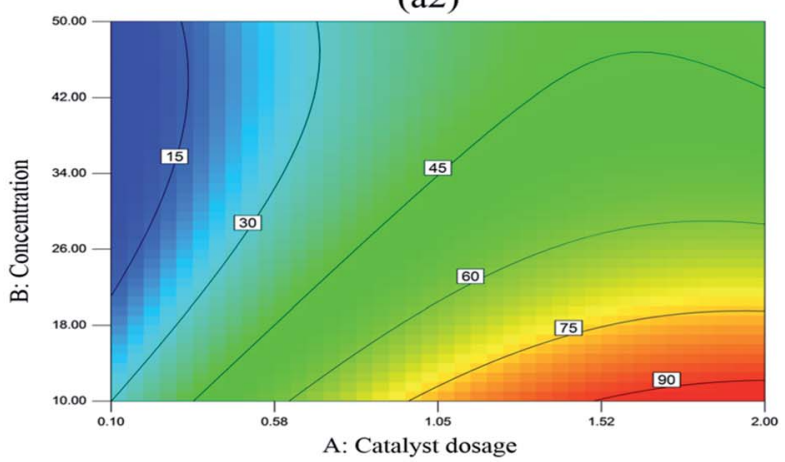

(b2)

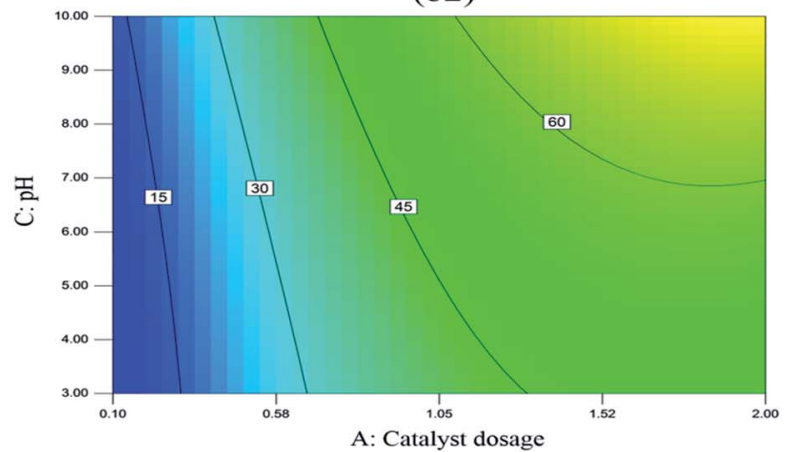

(c2)

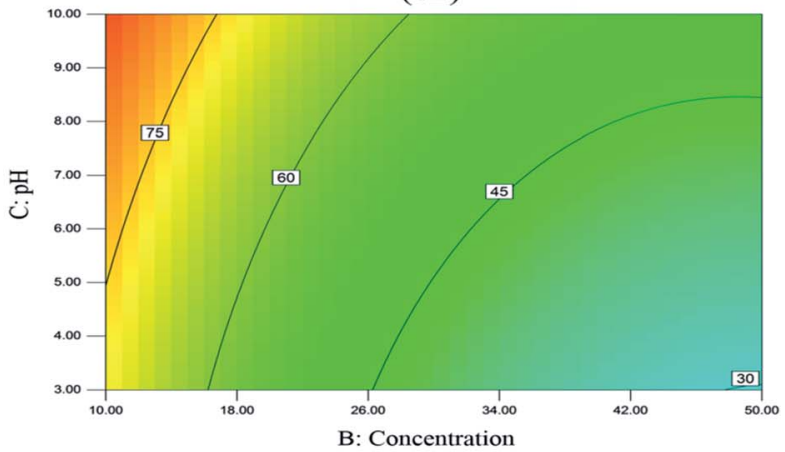

Fig. 5 Response surface graphs and contour plots (a1 and a2) showing the simultaneous effect of catalyst dosage and initial BPA concentration on BPA degradation, ( $\mathrm{b} 1$ and $\mathrm{b} 2$ ) the interaction between catalyst dosage and $\mathrm{pH}$ value, ( $\mathrm{c} 1$ and $\mathrm{c} 2$ ) the interaction between initial BPA concentration and $\mathrm{pH}$ value.

between two variables, while circular nature possibly indicates less prominent or negligible interactions. ${ }^{57}$ Hence, according to contour plot in Fig. 5(a2), catalyst dosage and concentration show prominent interaction on removal efficiency of BPA, which is in good agreement with statistically significant result in Table 2 and large magnitude of term $A B$ in eqn (3). As shown in two figures (Fig. 5(a1) and (a2)), BPA degradation is improved as expected by increasing catalyst dosage irrespective of initial BPA concentration at $\mathrm{pH}$ 6.5. It is likely related to more reaction sites resulting from more catalyst dosage. Significant improvement of BPA degradation is observed at low concentrations and the response is slightly increased at high BPA concentrations. For instance, when BPA concentration was $10 \mathrm{mg} \mathrm{L}^{-1}$, the removal efficiency increased from $26.4 \%$ to $93.3 \%$ with catalyst dosage ranged from 0.1 to $2 \mathrm{~g} \mathrm{~L}^{-1}$. However, once BPA concentration reached up to $50 \mathrm{mg} \mathrm{L}^{-1}$, BPA degradation varied from $6.9 \%$ to $45.0 \%$ at the same range of catalyst dosage. In addition, it can be seen that BPA decomposition is inhibited with the increase of BPA concentration irrespective of catalyst dosage. A possible explanation for the inverse relationship is limited reaction sites on the catalyst surface and catalyst deactivation by reactant and product accumulation. ${ }^{58}$

To investigate binary interaction of catalyst dosage and $\mathrm{pH}$ value at a constant concentration of BPA $\left(30 \mathrm{mg} \mathrm{L}^{-1}\right), 3 \mathrm{D}$ response surface and contour plots were shown in Fig. 5(b1) and (b2). It is clearly observed that compared to catalyst dosage, the influence of $\mathrm{pH}$ value on BPA decomposition is not remarkably significant. For instance, experimental response of BPA degradation increased from $26.9 \%$ to $48.0 \%$ when $\mathrm{pH}$ value changed from 3 to 10 with constant BPA concentration $\left(50 \mathrm{mg} \mathrm{L}^{-1}\right)$ and catalyst dosage $\left(1.05 \mathrm{~g} \mathrm{~L}^{-1}\right)$. It seems that for $\mathrm{Bi}_{7} \mathrm{O}_{9} \mathrm{I}_{3}$ catalyst, basic $\mathrm{pH}$ range is more beneficial to BPA degradation than 
acidic $\mathrm{pH}$, which is likely associated with more positive charge of photocatalyst surface in acidic $\mathrm{pH}$ range. ${ }^{58}$

Fig. 5(c1) and (c2) illustrated the interactive effect of BPA concentration and $\mathrm{pH}$ value, while catalyst dosage was kept constant at $1.05 \mathrm{~g} \mathrm{~L}^{-1}$ towards BPA degradation. The interaction is not significant due to a weak degree of curvature in 3D surface plot and circular contour plot. Overall, these graphs are feasible to understand individual and interaction effects of catalyst dosage, initial concentration of BPA and $\mathrm{pH}$ value on BPA degradation. Significant interaction between catalyst dosage and BPA concentration is observed during photocatalytic degradation, which is consistent with statistical analysis results.

\section{Conclusions}

A simple and fast microwave irradiation method was applied to synthesize $\mathrm{Bi}_{7} \mathrm{O}_{9} \mathrm{I}_{3}$ photocatalyst successfully. The assynthesized $\mathrm{Bi}_{7} \mathrm{O}_{9} \mathrm{I}_{3}$ exhibited superior photocatalytic activity over BiOI in the decomposition of BPA under xenon lamp illumination. Different micro/nano-structure, band gap energy and recombination rate of electron-hole pairs between $\mathrm{Bi}_{7} \mathrm{O}_{9} \mathrm{I}_{3}$ and $\mathrm{BiOI}$ are contributed to the different catalytic activity according to characterization results. BBD-RSM was performed to investigate individual and interaction influence of three independent variables including catalyst dosage, initial concentration of BPA and $\mathrm{pH}$ value on the removal efficiency of $\mathrm{BPA}$ using $\mathrm{Bi}_{7} \mathrm{O}_{9} \mathrm{I}_{3}$. Based on the proposed empirical quadratic model, ANOVA results and response surface analysis, catalyst dosage and BPA concentration show prominent interaction on removal efficiency of BPA and catalyst dosage is the most influential parameter. The findings not only indicate that the assynthesized $\mathrm{Bi}_{7} \mathrm{O}_{9} \mathrm{I}_{3}$ is promising candidates for practical application in wastewater treatment of BPA, but also show the potential of BBD-RSM to explore individual and interaction effects of different factors on removal efficiency of organic pollutants.

\section{Conflicts of interest}

There are no conflicts to declare.

\section{Acknowledgements}

This work was supported by grants from the National Natural Science Foundation of China (No. 41473096).

\section{References}

1 C. A. Staples, P. B. Dome, G. M. Klecka, S. T. Oblock and L. R. Harris, Chemosphere, 1998, 36, 2149-2173.

2 S. Flint, T. Markle, S. Thompson and E. Wallace, J. Environ. Manage., 2012, 104, 19-34.

3 S. Akbari, F. Ghanbari and M. Moradi, Chem. Eng. J., 2016, 294, 298-307.
4 N. Khalil, J. R. Ebert, L. Wang, S. Belcher, M. Lee, S. A. Czerwinski and K. Kannan, Sci. Total Environ., 2014, 470-471, 726-732.

5 L. Zhao, Y. F. Ji, D. Y. Kong, J. H. Lu, Q. S. Zhou and X. M. Yin, Chem. Eng. J., 2016, 303, 458-466.

6 I. A. Lang, T. S. Galloway, A. Scarlett, W. E. Henley, M. Depledge, R. B. Wallace and D. Melzer, J. Am. Med. Assoc., 2008, 300, 1303-1310.

7 C. Chang, L. Y. Zhu, Y. Fu and X. L. Chu, Chem. Eng. J., 2013, 233, 305-314.

8 Y.-R. Jiang, W. W. Lee, K.-T. Chen, M.-C. Wang, K.-H. Chang and C.-C. Chen, J. Taiwan Inst. Chem. Eng., 2014, 45, 207-218.

9 H. L. Chen, W. W. Lee, W.-H. Chung, H.-P. Lin, Y.-J. Chen, Y.-R. Jiang, W.-Y. Lin and C.-C. Chen, J. Taiwan Inst. Chem. Eng., 2014, 45, 1892-1909.

10 H. P. Lin, W. W. Lee, S. T. Huang, L. W. Chen, T. W. Yeh, J. Y. Fu and C. C. Chen, J. Mol. Catal. A: Chem., 2016, 417, 168-183.

11 H. F. Cheng, B. B. Huang and Y. Dai, Nanoscale, 2014, 6, 2009-2026.

12 Y. H. Lee, Y. M. Dai, J. Y. Fu and C. C. Chen, Mol. Catal., 2017, 432, 196-209.

13 S.-Y. Chou, W.-H. Chung, L.-W. Chen, Y.-M. Dai, W.-Y. Lin, J.-H. Lin and C.-C. Chen, RSC Adv., 2016, 6, 82743-82758.

14 Y. R. Jiang, H. P. Lin, W. H. Chung, Y. M. Dai, W. Y. Lin and C. C. Chen, J. Hazard. Mater., 2015, 283, 787-805.

15 Y. R. Jiang, S. Y. Chou, J. L. Chang, S. T. Huang, H. P. Lin and C. C. Chen, RSC Adv., 2015, 5, 30851-30860.

16 R. A. He, S. W. Cao, J. G. Yu and Y. C. Yang, Catal. Today, 2016, 264, 221-228.

17 S.-T. Huang, Y.-R. Jiang, S.-Y. Chou, Y.-M. Dai and C.-C. Chen, J. Mol. Catal. A: Chem., 2014, 391, 105-120.

18 X. Xiao, R. Hao, X. Zuo, J. M. Nan, L. S. Li and W. D. Zhang, Chem. Eng. J., 2012, 209, 293-300.

19 X. Xiao and W. D. Zhang, RSC Adv., 2011, 1, 1099-1105.

20 H. Liu, Y. Su, Z. Chen, Z. T. Jin and Y. Wang, J. Mol. Catal. A: Chem., 2014, 391, 175-182.

21 M. M. Xu, Y. L. Zhao and Q. S. Yan, Water Sci. Technol., 2015, 72, 2122-2131.

22 I. Tateishi, H. Katsumata, T. Suzuki and S. Kaneco, Catal. Lett., 2017, 147, 1503-1509.

23 S. Y. Chou, C. C. Chen, Y. M. Dai, J. H. Lin and W. W. Lee, RSC Adv., 2016, 6, 33478-33491.

24 H. Wang, J. Z. Xu, J. J. Zhu and H. Y. Chen, J. Cryst. Growth, 2002, 244, 88-94.

25 H. S. Pajaie and M. Taghizadeh, Synth. React. Inorg., Met.Org., Nano-Met. Chem., 2015, 46, 701-712.

26 N. A. A. Talib, F. Salam, N. A. Yusof, S. A. Alang Ahmad and Y. Sulaiman, RSC Adv., 2017, 7, 11101-11110.

27 S. L. Ferreira, R. E. Bruns, H. S. Ferreira, G. D. Matos, J. M. David, G. C. Brandao, E. G. da Silva, L. A. Portugal, P. S. dos Reis, A. S. Souza and W. N. dos Santos, Anal. Chim. Acta, 2007, 597, 179-186.

28 L. V. Candioti, M. M. De Zan, M. S. Camara and H. C. Goicoechea, Talanta, 2014, 124, 123-138.

29 V. Kumar Gupta, S. Agarwal, M. Asif, A. Fakhri and N. Sadeghi, J. Colloid Interface Sci., 2017, 497, 193-200. 
30 E. C. Catalkaya and F. Kargi, Chemosphere, 2007, 69, 485492.

31 S.-N. Nam, H. Cho, J. Han, N. Her and J. Yoon, Process Saf. Environ. Prot., 2018, 113, 10-21.

32 L. Ling, D. P. Zhang, J. Y. Fang, C. Fan and C. Shang, Chemosphere, 2017, 184, 417-428.

33 C. Sahoo and A. K. Gupta, J. Hazard. Mater., 2012, 215-216, 302-310.

34 X. Xiao, C. Liu, R. P. Hu, X. X. Zuo, J. M. Nan, L. S. Li and L. S. Wang, J. Mater. Chem., 2012, 22, 22840-22843.

35 M. Schmidt, H. Oppermann, H. Brückner and M. Binnewies, Z. Anorg. Allg. Chem., 1997, 623, 1945-1953.

36 W. W. Lee, C. S. Lu, C. W. Chuang, Y. J. Chen, J. Y. Fu, C. W. Siao and C. C. Chen, RSC Adv., 2015, 5, 23450-23463.

37 X. Xiao, C. L. Xing, G. P. He, X. X. Zuo, J. m. Nan and L. S. Wang, Appl. Catal., B, 2014, 148-149, 154-163.

38 X. F. Liu, X. Y. Xiong, S. P. Ding, Q. Q. Jiang and J. C. Hu, Catal. Sci. Technol., 2017, 7, 3580-3590.

39 K.-L. Li, W. W. Lee, C.-S. Lu, Y.-M. Dai, S.-Y. Chou, H.-L. Chen, H.-P. Lin and C.-C. Chen, J. Taiwan Inst. Chem. Eng., 2014, 45, 2688-2697.

40 M. Hino and T. Sato, Bull. Chem. Soc. Jpn., 1971, 44, 33-37.

41 M. Ma, Y. Zhang, W. Yu, H. Y. Shen, H. Q. Zhang and N. Gu, Colloids Surf., A, 2003, 212, 219-226.

42 Y. H. Xiang, P. Ju, Y. Wang, Y. Sun, D. Zhang and J. Q. Yu, Chem. Eng. J., 2016, 288, 264-275.

43 C. Liu and X. J. Wang, Dalton Trans., 2016, 45, 7720-7727.

44 A. J. Han, J. L. Sun, X. H. Lin, C. H. Yuan, G. K. Chuah and S. Jaenicke, $R S C$ Adv., 2015, 5, 88298-88305.

45 X. Xiao, M. L. Lu, J. M. Nan, X. X. Zuo, W. D. Zhang, S. M. Liu and S. B. Wang, Appl. Catal., B, 2017, 218, 398-408.
46 H. P. Lin, C. C. Chen, W. W. Lee, Y. Y. Lai, J. Y. Chen, Y. Q. Chen and J. Y. Fu, RSC Adv., 2016, 6, 2323-2336.

47 C. T. Yang, W. W. Lee, H. P. Lin, Y. M. Dai, H. T. Chi and C. C. Chen, $R S C$ Adv., 2016, 6, 40664-40675.

48 B. Z. Fang, A. Bonakdarpour, K. Reilly, Y. L. Xing, F. Taghipour and D. P. Wilkinson, ACS Appl. Mater. Interfaces, 2014, 6, 15488-15498.

49 B. Z. Fang, Y. L. Xing, A. Bonakdarpour, S. C. Zhang and D. P. Wilkinson, ACS Sustainable Chem. Eng., 2015, 3, 2381-2388.

50 S. Q. Fan, B. Z. Fang, J. H. Kim, J.-J. Kim, J.-S. Yu and J. Ko, Appl. Phys. Lett., 2010, 96, 063501.

51 B. Z. Fang, J. H. Kim, M.-S. Kim and J.-S. Yu, Acc. Chem. Res., 2013, 46, 1397-1406.

52 T. Y. Zhao, Z. Y. Liu, K. Nakata, S. Nishimoto, T. Murakami, Y. Zhao, L. Jiang and A. Fujishima, J. Mater. Chem., 2010, 20, 5095-5099.

53 F. Bagheban Shahri and A. Niazi, J. Magn. Magn. Mater., 2015, 396, 318-326.

54 X. Xiao, R. P. Hu, C. Liu, C. L. Xing, X. X. Zuo, J. M. Nan and L. S. Wang, Chem. Eng. J., 2013, 225, 790-797.

55 K. Yetilmezsoy, S. Demirel and R. J. Vanderbei, J. Hazard. Mater., 2009, 171, 551-562.

56 N. Biglarijoo, S. A. Mirbagheri, M. Ehteshami and S. M. Ghaznavi, Process Saf. Environ. Prot., 2016, 104, 150160.

57 J. F. Fu, Y. Q. Zhao and Q. L. Wu, J. Hazard. Mater., 2007, 144, 499-505.

58 N. Tafreshi, S. Sharifnia and S. Moradi Dehaghi, Process Saf. Environ. Prot., 2017, 106, 203-210. 